\title{
Cosmological test using the high-redshift detection rate of FSRQs with the Square Kilometer Array
}

\author{
Kyle Leaf ${ }^{1 \star}$ and Fulvio Melia ${ }^{2} \dagger$ \\ ${ }^{1}$ Department of Physics, The University of Arizona, AZ 85721, USA \\ ${ }^{2}$ Department of Physics, The Applied Math Program, and Department of Astronomy, The University of Arizona, AZ 85721, USA
}

Accepted XXX. Received YYY; in original form ZZZ

\begin{abstract}
We present a phenomenological method for predicting the number of Flat Spectrum Radio Quasars (FSRQs) that should be detected by upcoming Square Kilometer Array (SKA) SKA1-MID Wide Band 1 and Medium-Deep band 2 surveys. We use the Fermi Blazar Sequence and mass estimates of Fermi FSRQs, and $\gamma$-ray emitting Narrow Line Seyfert 1 galaxies, to model the radio emission of FSRQs as a function of mass alone, assuming a near-Eddington accretion rate, which is suggested by current quasar surveys at $z \gtrsim 6$. This is used to determine the smallest visible black hole mass as a function of redshift in two competing cosmologies we compare in this paper: the standard $\Lambda \mathrm{CDM}$ model and the $R_{\mathrm{h}}=c t$ universe. We then apply lockstep growth to the observed black-hole mass function at $z=6$ in order to devolve that population to higher redshifts and determine the number of FSRQs detectable by the SKA surveys as a function of $z$. We find that at the redshifts for which this method is most valid, $\Lambda \mathrm{CDM}$ predicts $\sim 30$ times more FSRQs than $R_{\mathrm{h}}=c t$ for the Wide survey, and $\sim 100$ times more in the Medium-Deep survey. These stark differences will allow the SKA surveys to strongly differentiate between these two models, possibly rejecting one in comparison with the other at a high level of confidence.
\end{abstract}

Key words: cosmology: large-scale structure of the universe, cosmology: observations, cosmology: theory, distance scale; galaxies: general

\section{INTRODUCTION}

The Square Kilometer Array (SKA) is a premier upcoming radio observatory, expected to provide a powerful probe of cosmological features (Square Kilometre Array Cosmology Science Working Group, 2018, hereafter SKA CSWG 2018). Phase 1 of the SKA, constituting roughly $10 \%$ of the design collecting area, is expected to be constructed from 2020 to 2025 . Of principal interest for the work reported in this paper, SKA will significantly enhance our ability to detect high-redshift, radio-loud quasars. The primary goal of this analysis is to calculate the number of such sources that ought to be detectable by Phase 1 of the SKA in two competing models - standard $\Lambda \mathrm{CDM}$ and the $R_{\mathrm{h}}=c t$ universe - thereby providing a new test of the background cosmology.

The current standard model has been quite successful in describing many evolutionary aspects of the Universe, though we have begun to see growing tension between its predictions and several key observations. For example, the

^ kyleaf@email.arizona.edu

$\dagger$ John Woodruff Simpson Fellow. E-mail:

fmelia@email.arizona.edu

(C) 2019 The Authors
Hubble constant measured with Planck (Planck Collaboration 2018) disagrees with the locally measured value at over $4 \sigma$ significance (Riess et al. 2018). A very different type of measurement, based on the angular-diameter distance, shows that the peak of this integrated measure also disagrees with our expectation from $\Lambda$ CDM (Melia \& Yennapureddy 2018a). All in all, comparative tests between the standard model and the alternative picture we shall consider in this paper have now been completed using over 25 different kinds of data. The standard model has been disfavoured by the observations in all of these cases (see, e.g., Table 2 in Melia 2018). We are therefore motivated to at least consider modifications to the background cosmology, perhaps eventually even replacing it, in order to determine whether different physics may better describe the growing observational evidence.

The SKA, with its unprecedented capabilities, is poised to probe the intermediate to high-redshift radio universe with greater sensitivity than any instrument before it. In our previous study of high-redshift active galaxies (Fatuzzo \& Melia 2017), we analyzed the high-redshift quasar detection rate based on their X-ray emission in order to predict the various quasar counts to be compiled by the upcom- 
ing eROSITA and ATHENA observatories. While $R_{\mathrm{h}}=c t$ and $\Lambda \mathrm{CDM}$ are very similar in describing the local universe, their predictions diverge towards higher redshifts. Of direct relevance to this study are the differences in the two models between the comoving volume and age of the Universe as functions of redshift. In our previous work, the combination of those factors, based on the existing observations of quasars at $z \sim 6$, resulted in predictions of $\sim 0.16 \mathrm{X}$ ray emitting quasars detectable by ATHENA at $z \sim 7$ in $R_{\mathrm{h}}=c t$, compared to $\sim 160$ such objects in $\Lambda$ CDM. Such a stark difference will be easily measurable by these upcoming ATHENA observations. In this paper, we extend this work significantly by considering the radio-loud quasar population, which will allow us to probe the Universe even more deeply with SKA. Indeed, we shall demonstrate that SKA1's medium-deep survey has the potential of detecting jetted AGN with central black-hole masses as small as $M \sim 10^{6} M_{\odot}$ out to a redshift of $z=9$.

But our chief concern here is not only to estimate how many new radio-loud quasars we can hope to see with SKA in the remote Universe; we shall argue that their detection rate will be strongly dependent on the assumed background cosmology - offering us the interesting prospect of using these counts as a powerful test of the cosmology based on techniques that are quite different from the more conventional, integrated measures, such as luminosity distances and the Hubble parameter. We shall seek to characterize the radio emission profile of a specific class of high-redshift active galaxies and evolve the population known at $z \sim 6$ to higher redshifts, assuming standard Eddington-limited accretion, in order to predict their detection rate as a function of $z$ for each cosmological model.

In $\S$ II, we shall discuss the radio emission characteristics of these high-redshift quasars, and describe the methods used to estimate their growth and evolution with redshift in each of these models in $\S$ III. To do this, we shall also summarize here the key differences between $R_{\mathrm{h}}=c t$ and $\Lambda \mathrm{CDM}$ to the extent that these variations affect the AGN population synthesis. We shall present and discuss our results in $\S \mathrm{IV}$, and finally conclude in $\S \mathrm{V}$.

\section{THE RADIO EMISSION OF HIGH-Z QUASARS}

As of today, approximately half of the high- $z$ (i.e., $z>5.5$ ), radio-loud quasars have been identified as Flat Spectrum Radio Quasars (FSRQs), with the remaining half showing steeper spectra similar to Faranov-Riley II (FR-II) galaxies (Coppejans et al. 2016). FSRQs are generally considered to be active galactic nuclei (AGN) with powerful relativistic jets in a configuration such that the jet is aligned towards the observer, commonly referred to as a blazar (Beckmann \& Shrader 2012). Blazars also include BL Lacertae objects (see, for example, the review papers by Antonucci 1993, and Sikora \& Madejski 2001). These, however, have not been detected at high redshifts. As discussed in Ghisellini et al. (2009a), another distinguishing feature between BL Lacs and FSRQs is that the former have significantly lower accretion rates while, in the high-redshift $(z>5.5)$ Universe, the central black holes of FSRQs are found to be emitting at or near the Eddington luminosity (Willott et al. 2010). Cir- cumstantial evidence, largely based on superluminal motion (Hovatta et al. 2009), suggests that the relativistic jets of FSRQs have bulk Lorentz factors $\Gamma \sim 5-15$ (Ghisellini \& Sbarrato, 2016).

Assuming a random distribution of AGN orientation, the probability of seeing any given jetted (and thus, radioloud) quasar in its blazar configuration is $\sim 1 / 2 \Gamma^{2}$, implying roughly a range of 1 in 50 to 1 in 450 (Volonteri et al. 2011). While this low blazar fraction is consistent with observations of AGNs at low $z$ (i.e., $z<3$ ), it stands in sharp contrast to the roughly 1 in 2 chance of a radio-loud, $z>4.5$ AGN being an FSRQ (Coppejans et al. 2016). Several mechanisms have been proposed to help explain this observation (Fabian et al. 2014; Ghisselini \& Sbarrato 2016; Wu et al. 2017), but analyses have been inconclusive. These proposals generally suggest that the fraction of jetted AGNs at high redshift that appear as FSRQs are the same as their lower-redshift counterparts, but that those without the jet preferentially directed at the observer are not observed strongly in the radio band.

The total spectral energy distribution (SED) across all wavebands for an AGN depends on a large number of physical phenomena. The more commonly included components of quasar emission are: a relativistic jet emitting in the radio (Urry \& Padovani, 1995) and, sometimes, in X-rays and $\gamma$ rays via inverse Compton scattering (Melia \& Königl 1989); a thermally-radiating accretion disk emitting predominantly at UV wavelengths (Shakura \& Sunyaev 1973); a hot corona emitting primarily in X-rays (Lusso \& Risalti 2016; Fatuzzo \& Melia 2017), and an obscuring torus (Krolik \& Begelman 1986); as well as any radiation from the stars in the rest of the galaxy. Roughly $8.1_{-3.2}^{+5.0} \%$ (Bañados et al. 2015) of the AGNs at high redshift are known to emit strongly at radio frequencies. Disentangling all of the various contributions to the overall SED can be quite challenging, especially given the diversity of AGNs sub-samples distinguished primarily upon the dominant contributions from these many components. Rather than constructing a model based on this comprehensive emission picture, we therefore take a more phenomenological approach, analyzing one specific class of quasar that ought to be the most relevant for their detection by the upcoming SKA surveys.

Our empirical approach is geared towards modeling the appearance of high- $z$ FSRQs in order to estimate the number of such objects that will be visible to SKA. In spite of the different population statistics referenced previously, FSRQs at high redshift appear to have very similar SEDs to those at lower redshift. For instance, the confirmed highestredshift FSRQ to date, J0906+6930 at $z=5.47$, has an observed SED similar to lower- $z$ FSRQs (An \& Romani 2018). It is therefore reasonable to suppose that the large suite of lower-redshift FSRQ observations may be used to characterize the radio emission profile of such objects even at higher redshifts.

Ghisellini et al. (2017) presented the so-called blazar sequence based on the Fermi observations compiled in Ackermann et al. (2015), an attempt to characterize the average SED of blazars separated into $\gamma$-ray luminosity bins. That work included separate analyses of BL Lac objects and FSRQs. As no BL Lacs have been observed at high redshifts, we here limit ourselves to the FSRQ data, in which the blazars are grouped into five bins based on their $\gamma$-ray luminosity, 
$L_{\gamma}$. These authors included the observation that the overall SED of FSRQs does not change significantly with changing $L_{\gamma}$, instead generally differing only in terms of emitted power. Furthermore, all FSRQs in each bin were found to be consistent with a radio spectral index $\alpha_{R} \approx-0.1$, a parameter that was then held fixed in the fitting. The results presented in Ghisellini et al. (2017) are based on an assumed $0.7=h=\Omega_{\Lambda}$ cosmology. Therefore, in order to extract the results relevant for $R_{\mathrm{h}}=c t$, we recalibrate the inferred luminosity using the ratio $\left(d_{L}^{R_{\mathrm{h}}=c t} / d_{L}^{\Lambda \mathrm{CDM}}\right)^{2}$ to account for the differences between these models, in terms of the luminosity distance $d_{L}$. For objects in the 3LAC catalog, the inferred luminosities are $\sim 15-19 \%$ smaller in $R_{\mathrm{h}}=c t$ than in the fiducial $\Lambda$ CDM model.

For each of the five phenomenological SEDS from Ghisellini et al. (2017), we determine the $\gamma$-ray luminosity over the Fermi band $(0.1 \mathrm{GeV}-100 \mathrm{GeV})$ for each, and compare these results to the catalog of bright Fermi blazars in Ghisellini et al. (2010). This sample includes 22 FSRQs with both observed $\gamma$-ray luminosities and published central black-hole mass estimates. The masses are determined by associating an optical-UV bump in each object's SED with direct emission from the accretion disk. Ghisellini et al. (2009b) and Ghisellini et al. (2010) suggest that this method is accurate to within a factor of 2 . The $v F_{v}$ peak of the disk luminosity is used to determine the maximum temperature of the accretion disk, which scales as $T_{\max } \propto\left(\lambda_{\mathrm{Edd}}\right)^{1 / 4} M^{-1 / 4}$, where $\lambda_{\text {Edd }}$ is the Eddington factor (i.e., the ratio of luminosity to its Eddington value). Furthermore, the total observed flux from the accretion disk scales as $\lambda_{\mathrm{Edd}} M / d_{L}^{2}$. Therefore, each mass determined by Ghisellini et al. (2010) must be multiplied by the factor $d_{L}^{R_{\mathrm{h}}=c t} / d_{L}^{\Lambda \mathrm{CDM}}$ in order to get the appropriate inferred mass for the $R_{\mathrm{h}}=c t$ universe. The inferred Eddington ratio in $R_{\mathrm{h}}=c t$ changes by the same factor.

These masses recorded in Ghisellini et al. (2010) (quoted as $\left.\log _{10}\left[M / M_{\odot}\right]\right)$ range from 8.17 to 9.78 , with luminosities $\left(\log _{10}\left[L_{\gamma} / \mathrm{erg} \mathrm{s}^{-1}\right]\right)$ from 45.93 to 49.10 , at redshifts from $z=0.213$ to 2.19 . Using the fitted SEDs from Ghisellini et al. (2010), we estimate the average disk luminosity of these observed FSRQs as a ratio of the Eddington limit, yielding a value $\approx 0.22$, providing some evidence that the accretion rates are generally high. Since the lowest $\gamma$-ray bin in the Fermi blazar sequence of FSRQs falls in the range $10^{44}-10^{45}$ $\mathrm{erg} \mathrm{s}^{-1}$, below the range of the bright Fermi blazars, we need an additional sample of high accretion rate active galaxies in order to associate that bin with a central black-hole mass.

With high redshift FSRQs known to have generally high accretion rates, in order to associate the lower $\gamma$-ray luminosity bins of the blazar sequence with some fiducial central black-hole mass, we must select those objects among this category that themselves have a high accretion rate, but also lower luminosity. Gamma-ray emitting Narrow Line Seyfert 1 galaxies ( $\gamma$-NLSy1) are radio-loud active galaxies characterized by flat radio spectra, exhibiting a core-jet structure, high brightness temperature, and apparent superluminal motion. Indeed, the broad-band SEDs of $\gamma$-NLSy1 resemble those of FSRQs, but merely less powerful (Paliya et al. 2013). More recent analyses of these objects (Paliya et al. 2018; Paliya et al. 2019) have further established a link between $\gamma$-NLSy1s and FSRQs. For example, attempts to fit their SEDs have shown that their jets have bulk Lorentz

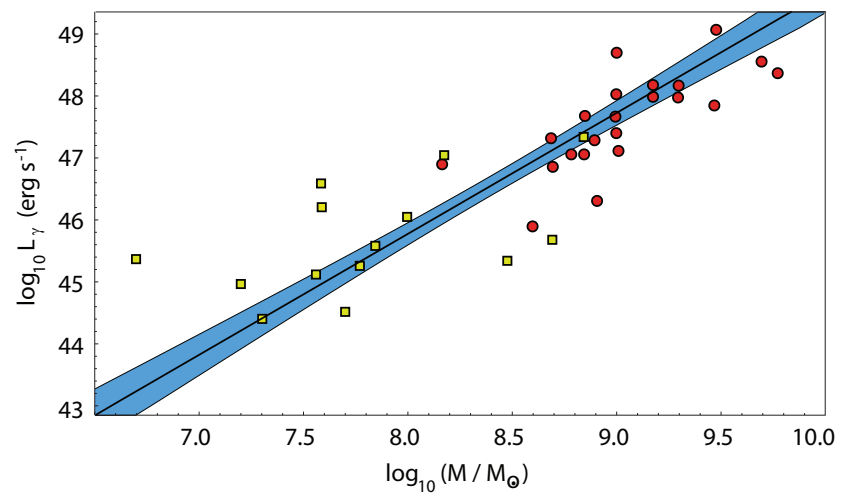

Figure 1. $\gamma$-ray luminosity $\log _{10}\left(L /\left[\mathrm{erg} \mathrm{s}^{-1}\right]\right)$ vs. central black-hole mass $\log _{10}\left(M / M_{\odot}\right)$ in $\Lambda$ CDM for bright Fermi FSRQs (red circles) (Ghisellini et al. 2009), and the $14 \gamma$-NLSy1s (yellow squares) with measured $\gamma$-ray luminosity and central black-hole mass in the context of $\Lambda$ CDM. (Paliya et al. 2018, 2019). The line shows the best-fit power law, along with its estimated uncertainty (blue swath) based on standard error propagation.

factors in the range $7<\Gamma<17$, comfortably within the usual estimated factors of FSRQs, as noted above.

For our phenomenological model construction, we therefore use the 14 known $\gamma$-NLSy1's included in Paliya et al.'s (2018) sample. We take their reported $\gamma$-ray luminosity and the mass estimates derived from optical spectroscopy recorded in Paliya et al. (2019), and citations recorded therein. In $R_{\mathrm{h}}=c t$, these mass estimates are adjusted in the same manner as FSRQs. This sample includes reported luminosities $\log _{10}\left(L_{\gamma} /\left[\mathrm{erg} \mathrm{s}^{-1}\right]\right)$ from 44.4 to 47.4 , and estimated black-hole masses from $10^{6.7}-10^{8.85} M_{\odot}$. Together with the highly luminous Fermi FSRQs from Ghisellini et al. (2010), we therefore have a sample of 36 active galaxies with observed gamma-ray luminosities, mass estimates, and a generally high estimated inferred accretion rate, consistent with those $z>5.5$ quasars observed to date. Figure 1 shows the $\gamma$-ray luminosity plotted against the central blackhole mass for this sample, as well as our best-fit (power-law) function to describe their correlation.

This best-fit power law is found with linear regression using the logrithms of the estimated central black-hole mass and luminosity, which is taken as the independent variable. The error in the mean mass is estimated according to

$\delta \mu\left(l_{\gamma}\right)=\frac{\sigma_{\mu}}{\sqrt{n}} \times \sqrt{1+\frac{l_{\gamma}-\left\langle l_{\gamma}\right\rangle}{\sigma_{l_{\gamma}}^{2}}}$,

where $\mu \equiv \log _{10} m, l_{\gamma} \equiv \log _{10}\left(L_{\gamma} /\left[\mathrm{erg} \mathrm{s}^{-1}\right]\right), n$ is the number of observations, $\sigma_{\mu}$ is the square root of the reduced $\chi^{2} /$ dof, $\sigma_{l_{\gamma}}^{2}$ is the population variance of $l_{\gamma}$, and $\left\langle l_{\gamma}\right\rangle$ is the mean of all $l_{\gamma}$ values. Table 1 shows the five SED bins from Ghisellini et al. (2017), the $\gamma$-ray luminosity found by integrating the SED from $0.1 \mathrm{GeV}$ to $100 \mathrm{GeV}$ (we point out that integrating from $0.1 \mathrm{GeV}$ to $300 \mathrm{GeV}$ instead does not change the numerical result by more than $3.7 \%$ or $0.016 \mathrm{dex}$ ), and the mass we associate with each luminosity in both models. The errors in the masses are simply those described by Equation (1) for the given $L_{\gamma}$.

The Fermi Blazar Sequence of Ghisellini et al. (2017) 
Table 1. Fermi blazar sequence bins, their $\gamma$-ray luminosities, and their fitted masses.

\begin{tabular}{lcccc}
\hline \hline & & & \\
$\begin{array}{l}\log _{10} L_{\gamma} \\
\left(\mathrm{erg} \mathrm{s}^{-1}\right)\end{array}$ & $\begin{array}{c}\log _{10} L_{\gamma}^{\Lambda \mathrm{CDM}} \\
\left(\mathrm{erg} \mathrm{s}^{-1}\right)\end{array}$ & $\begin{array}{c}\log _{10} M^{\Lambda \mathrm{CDM}} \\
\left(M_{\odot}\right)\end{array}$ & $\begin{array}{c}\log _{10} L_{\gamma}^{R_{\mathrm{h}}=c t} \\
\left(\mathrm{erg} \mathrm{s}^{-1}\right)\end{array}$ & $\begin{array}{c}\log _{10} M^{R_{\mathrm{h}}=c t} \\
\left(M_{\odot}\right)\end{array}$ \\
\hline & & & & \\
$48<L$ & 48.27 & $9.28 \pm 0.10$ & 48.19 & $9.24 \pm 0.10$ \\
$47<L<48$ & 47.43 & $8.84 \pm 0.07$ & 47.34 & $8.80 \pm 0.07$ \\
$46<L<47$ & 46.54 & $8.39 \pm 0.07$ & 46.64 & $8.34 \pm 0.07$ \\
$45<L<46$ & 45.59 & $7.90 \pm 0.10$ & 45.59 & $7.87 \pm 0.10$ \\
$44<L<45$ & 44.59 & $7.39 \pm 0.14$ & 44.59 & $7.36 \pm 0.14$ \\
& & & & \\
\hline \hline
\end{tabular}

models their complete non-thermal SED in terms of two broad humps and a flat radio spectrum. Beginning at the lowest energies, the sequence starts with the flat radio segment from arbitrarily low frequencies up to some cutoff $v_{t}$. Above this, the single power law connects with two smoothly broken power laws. The lower frequency power law is associated with the synchrotron flux, and the higher frequency is associated with inverse Compton flux. Altogether, there are 11 parameters determined by observations. The first set contains three typical frequencies: $v_{t}$ is where the flat radio spectrum ends; $v_{S}$ is the peak of the synchrotron spectrum; and $v_{C}$ is the peak of the high-energy spectrum. Second, there are two cut-off frequencies at which the power laws start to exhibit exponential decay. These are $v_{\mathrm{Cut}, \mathrm{S}}$, and $v_{\mathrm{Cut}, \mathrm{C}}$. Third are the four power-law indices: $\alpha_{R}$ for the radio band; $\alpha_{1}$ connecting $v_{t}$ and $v_{S} ; \alpha_{2}$ giving the downward slope after both the $v_{S}$ and $v_{C}$ peaks, and $\alpha_{3}$ yielding the upward slope toward the $v_{C}$ peak. The remaining two parameters are the luminosities at the two peaks, which are included as $v_{S} L\left(v_{S}\right)$ (the luminosity at the Synchrotron peak) and ratio of the $v L_{v}$ Compton and synchrotron luminosities, known as the Compton Dominance (CD).

Having associated each Fermi blazar sequence SED with a central black hole of some mass undergoing Eddingtonlimited accretion, we interpolate the parameters characterizing the five models, resulting in a phenomenological SED for FSRQs as a function of $M$. Following Ghisellini et al. (2017), we hold the radio spectral index constant at $\alpha_{R}=-0.1$ throughout this work. For this interpolation, we additionally hold $v_{\text {cut, } \mathrm{S}}$ and $v_{\text {cut, }} \mathrm{C}$ constant at $10^{16} \mathrm{~Hz}$ and $10^{27} \mathrm{~Hz}$, respectively, as they were inferred to be relatively constant across the sequence models and do not significantly affect the overall SED. The indices $\alpha_{1}$ and $\alpha_{2}$ show no significant evolution with luminosity bin, so we hold them fixed at 0.5 and 1.43, respectively, while $\alpha_{3}$ is fitted by a linear relation in $\log M$. The frequencies $v_{t}$ and $v_{S}$ are fitted by linear functions in $\log M$ for the lower bins, and both are held at $10^{12} \mathrm{~Hz}$ for the brightest two bins. The frequency $v_{C}$ is fitted with a quadratic interpolation between the lowest three bins, and held fixed for $M>10^{8.23} M_{\odot}$, while $v_{S L}$ and CD are interpolated with quartic polynomial functions of $\log M$.

The final interpolated blazar sequence may be used to estimate the emission profile of any FSRQ within any waveband, from radio to $\gamma$-rays. We use the mass-dependent SED function to find a simple relationship between an FSRQ's central black-hole mass and its radio luminosity, which we then extrapolate downward to smaller masses. To do this, we switch from the full interpolated SED to a simplified relationship covering only the radio emission below $10^{7.39} M_{\odot}$ in $\Lambda \mathrm{CDM}$, and $10^{7.36} M_{\odot}$ in $R_{\mathrm{h}}=c t$. We fit a power law to the three lower-luminosity SEDs, and find a relationship $L_{R} \propto M^{1.55}$ in $\Lambda \mathrm{CDM}$, and $L_{R} \propto M^{1.56}$ in $R_{\mathrm{h}}=c t$. Even at a redshift of 7 , an FSRQ matching the SED in the lowest bin of the blazar sequence would have a radio flux density in the SKA1 band of $300 \mu \mathrm{Jy}$ in $\Lambda \mathrm{CDM}$, and $269 \mu \mathrm{Jy}$ in $R_{\mathrm{h}}=c t$, significantly above the $22.8 \mu \mathrm{Jy}$ flux limit of the Wide Band 1 survey (SKA CSWG 2018), requiring some extrapolation to lower masses in order to estimate the detection rate of smaller-though more numerous-AGNs.

\section{CALCULATIONS}

Now that the blazar radio emission may be estimated as a function of black-hole mass, we use the distribution from Willott et al. (2010) to establish (i.e., normalize) the mass function at a known redshift. Following Fatuzzo \& Melia (2017), we begin with the observed black-hole mass function at $z=6$, and devolve the entire population in lockstep towards higher redshifts, with the assumption that all quasars at $z=6$ are undergoing Eddington-limited accretion, with a duty cycle very close to 1 . This growth with a fiducial efficiency of $10 \%$ corresponds to an $e$-folding time of $t_{\text {Edd }} \approx 45$ Myr (known as the Salpeter time; see, e.g., Melia 2013). The overall growth rate of quasars at high redshifts is therefore highly dependent on the cosmology, given that the predicted timeline $t(z)$ changes considerably between models.

The two competing cosmological models we consider here are the standard flat $\Lambda$ CDM model and the $R_{\mathrm{h}}=c t$ universe. As noted earlier, while $\Lambda \mathrm{CDM}$ continues to account reasonably well for a broad range of data, several significant tensions continue to grow. For instance, the final results of the Planck mission (Planck Collaboration 2018) suggest a Hubble Constant statistically incompatible (now over $4 \sigma$ ) with that inferred by Hubble Space Telescope data (Riess et al. 2018). In addition - and a primary motivator for this work-Weigel at al. (2015) have estimated that, in the context of $\Lambda \mathrm{CDM}, \sim 20 \mathrm{AGNs}$ should have been observed at $z>5$ in the Chandra Deep Field South survey, while none were identified - strongly favouring $R_{\mathrm{h}}=c t$ over the standard model (Fatuzzo \& Melia 2017). Though possible sys- 
tematic effects may be at least partially responsible for this, at face value we estimate that this negative result constitutes a roughly $\sim 20 / \sqrt{20}$ effect, i.e., another $\sim 4 \sigma$ disparity between the predictions of the standard model and actual observations. And more recently, Wang et al. (2018) derived the quasar luminosity function at $z \sim 6.7$, which shows a significantly steeper reduction of the comoving quasar number density as a function of redshift than earlier estimates by Fan et al. (2001) based on $\Lambda$ CDM. Of utmost importance in calculating this change in quasar number density as a function of redshift is the amount of time elapsed from one redshift to another, which is strongly dependent on the presumed cosmological model.

For example, $z=6$ corresponds to $\approx 0.92$ Gyr after the Big Bang in flat $\Lambda$ CDM with parameters $\Omega_{\mathrm{m}}=0.3, H_{0}=70$ $\mathrm{km} \mathrm{s}^{-1} \mathrm{Mpc}^{-1}$. We adopt this parametrization for the entire analysis. By comparison, it is $\approx 2$ Gyr in $R_{\mathrm{h}}=c t$ with the same Hubble constant (which is the only free parameter in this model). The $R_{\mathrm{h}}=c t$ universe is a FriedmanLemaitre-Robertson-Walker (FLRW) cosmology with zero active mass, $\rho+3 p=0$ (Melia 2003, 2007, 2016, 2017, 2018; Melia \& Abdelqader 2009; Melia \& Shevchuk 2012). As noted in the introduction, it has accounted for the observations better than $\Lambda \mathrm{CDM}$ in comparative tests, including such notable cases as cosmic chronometers (Melia \& Maier 2013; Leaf \& Melia 2017; Melia \& Yennapureddy 2018b); strongly-lensed galaxies (Leaf \& Melia 2018; Yennapureddy \& Melia 2018); and measurements of the maximum angular-diameter distance (Melia 2018; Melia \& Yennapureddy 2018a). The different timeline in $R_{\mathrm{h}}=c t$ makes it especially attractive when considering the growth rates of black-hole seeds into supermassive black holes, a topic addressed in detail in Melia (2013) and Melia \& McClintock (2015). In $\Lambda$ CDM, the time elapsed between $z=7$ and $z=6$ is $167 \mathrm{Myr}$, compared with $249 \mathrm{Myr}$ in $R_{\mathrm{h}}=c t$. With a $\sim 45$ Myr e-folding time associated with Eddington-limited growth based on $10 \%$ efficiency (see, e.g., Fatuzzo \& Melia $2017), R_{\mathrm{h}}=c t$ black holes could grow by a factor of $\sim 150$ in that redshift range, while they would only have had time to grow by a factor of $\sim 28$ in $\Lambda$ CDM. This choice, that all of the blazars of interest are experiencing Eddington-Limited growth is motivated by the inferred Eddington ratio of high$\mathrm{z}$ quasars (such as those in the Wilott et al. 2010 sample). Thus, with the greater elapsed time, black holes at $z>6$ are naturally expected to have been significantly smaller in $R_{\mathrm{h}}=c t$ (for a given redshift) than in the concordance $\Lambda \mathrm{CDM}$ model.

The extrapolation to lower masses brings its own issues with the Willott et al (2010) black-hole mass function, however. This distribution is well constrained only for masses in the range $10^{8} M_{\odot} \lesssim M \lesssim 3 \times 10^{9} M_{\odot}$. As we shall demonstrate below, the Willott et al. mass function, when extrapolated to lower masses, may significantly overestimate the number of active galaxies. We begin with the black-hole mass function observed at $z=6$, then devolve objects to higher redshift by assuming all central black holes were accreting at the Eddington rate with an efficiency of 0.1. Analytically, a black hole accreting with efficiency $\epsilon_{r}$, and with a bolometric luminosity of some fraction of the Eddington luminosity $\lambda_{\text {Edd }} \equiv L_{\mathrm{Bol}} / L_{\text {Edd }}$ will have a mass-growth rate of

$\dot{M}=\frac{\left(1-\varepsilon_{r}\right)}{\varepsilon_{r}} \frac{\lambda_{\mathrm{Edd}} L_{\mathrm{Edd}}}{c^{2}}=\frac{\left(1-\varepsilon_{r}\right)}{\varepsilon_{r}} \frac{\lambda_{\mathrm{Edd}}}{t_{\mathrm{Edd}}} M$.

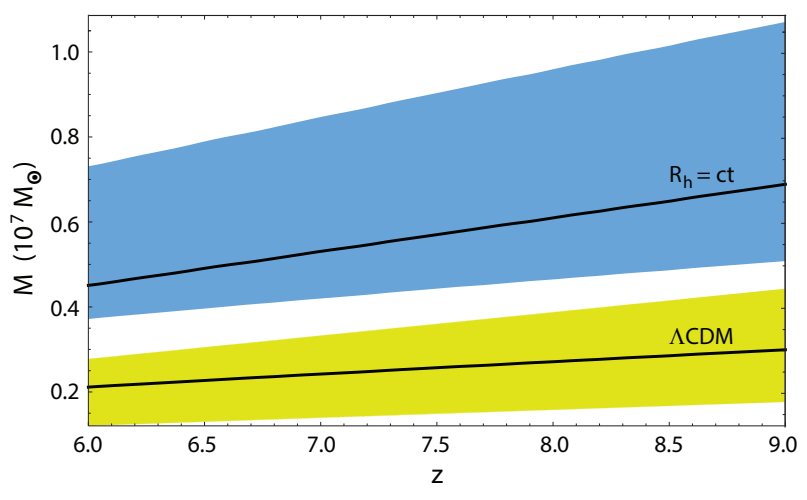

Figure 2. Smallest visible blazar in the SKA1 Wide Band 1 survey, along with our estimated errors: blue for $R_{\mathrm{h}}=c t$, and yellow for $\Lambda$ CDM (with $\Omega_{\mathrm{m}}=0.3$ ). The difference is due to the different radio luminosities associated with each mass, and the difference in luminosity distance for each model. The radio luminosity from the Fermi blazar sequence is extrapolated to lower masses with a power law.

Integrating with respect to time gives the mass of the black hole as a function of time,

$M\left(t_{z}\right)=M_{0} e^{\frac{\left(1-\varepsilon_{r}\right)}{\varepsilon_{r}} \frac{\lambda_{\mathrm{Edd}}}{t_{\mathrm{Edd}}}\left(t_{z}-t_{\mathrm{obs}}\right)}$,

where $t_{\mathrm{obs}}$ is the age of the universe at the observed redshift, and $t_{z}$ is the age of the universe at redshift $z$.

The black-hole mass function may then be written,

$\Phi_{M}(M, z)=\Phi_{z=6}\left[M e^{\frac{\left(1-\varepsilon_{r}\right)}{\varepsilon_{r}} \frac{\lambda_{\text {Edd }}}{t_{\text {Edd }}}\left(t_{6}-t_{z}\right)}\right]$,

where $t_{6}$ and $t_{z}$ are the ages of the Universe, respectively, at redshifts 6 and $z$, and $1-\epsilon_{r}$ gives the fraction of infalling material accreted onto the central black hole. We follow Willott et al. (2010) by setting a fiducial $\lambda_{\text {Edd }}=1, \epsilon_{r}=0.1$ and adopt $t_{\text {Edd }}=0.045$ Gyr, corresponding to the Salpeter ( $e$-folding) time of 45 Myr.

The normalization $\Phi_{z=6}$ in this expression is the fitted Schechter mass function

$\Phi_{z=6}(M)=\Phi_{0}\left(\frac{M}{M^{*}}\right)^{\beta} e^{-M / M^{*}}$

from Willott et al. (2010), with the best-fit parameters $\Phi_{0}=1.23 \times 10^{-8} \mathrm{Mpc}^{-3} \mathrm{dex}^{-1}, M^{*}=2.24 \times 10^{9} M_{\odot}$, and $\beta=-1.03$. This mass function is best constrained for the $z=6$ quasars with $M>10^{8} M_{\odot}$. Note, however, that no formal error estimation was made in that paper. We do not attempt to estimate that error here either, but infer that it would not be significant to our final results compared with the error in the high- $z$ radio-loud fraction and the errors in associating an FSRQ central black-hole mass with a SED.

Over the frequency range $(0.35,1.05) \mathrm{GHz}$, the SKA1Wide survey will probe 20,000 square degrees of sky (representing $\sim 48 \%$ coverage) to a sensitivity of $22.8 \mu \mathrm{Jy}$ (SKA CSWG 2018). A $10^{9} M_{\odot}$ FSRQ at redshift 6 is expected to have a flux $\sim 0.1 \mathrm{Jy}$ in this range, making it easily detectable. Likewise, the SKA1-Medium-Deep survey will probe from $0.95 \mathrm{GHz}$ to $1.75 \mathrm{GHz}$ down to its sensitivity limit of $8.2 \mu \mathrm{Jy}$ over 5,000 square degrees of sky (SKA CSWG 2018). We can therefore determine the smallest (in terms of black-hole 


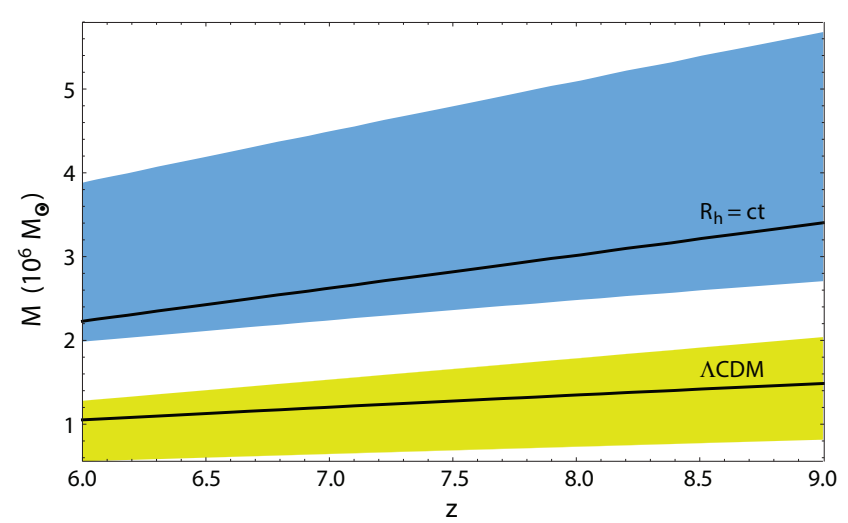

Figure 3. Same as figure 2, except for the SKA1 Medium-Deep Band 2 survey.

mass) AGN as a function of redshift visible to the SKA1 surveys for each cosmological model. The SED is shifted out of the AGN's rest frame by the required factor of $1+z$, such that the luminosity over SKA's observable band is

$L_{R}(M)=\int_{v_{1}(z+1)}^{v_{2}(z+1)} L_{R, v}(M) d v$.

The flux density in Jy over the observable band is then

$F_{R}(M, z)=\frac{L_{R}(M)}{\left(v_{2}-v_{1}\right) 4 \pi d_{L}^{2}(z)}$,

where $d_{L}(z)$ is given in Equations (7) and (8) as a function of $z$, and $v_{1}$ and $v_{2}$ are the upper and lower frequencies of the observable band. In $\Lambda \mathrm{CDM}$, we have

$d_{L}^{\Lambda \mathrm{CDM}}=\frac{c}{H_{0}}(1+z) \int_{0}^{z} \frac{d u}{\sqrt{\Omega_{\mathrm{m}}(1+u)^{3}+\Omega_{\mathrm{r}}(1+u)^{4}+\Omega_{\Lambda}}}$,

while in $R_{\mathrm{h}}=c t$ it takes the simpler form

$d_{L}^{R_{\mathrm{h}}=c t}=\frac{c}{H_{0}}(1+z) \ln (1+z)$

Next, we find the central black-hole mass that satisfies the equality $F_{\text {lim }}=F_{R}(M, z)$, where $F_{\text {lim }}$ is the flux limit of SKA. The error in these masses is the same as the error associated with each luminosity from Equation (1). This error is represented by the colored swaths in figures 2 and 3 .

Since the e-folding growth time is $\sim 45 \mathrm{Myr}$, a $10^{8} M_{\odot}$ object at $z=6$ would have grown from a $5 \times 10^{6} M_{\odot}$ black hole (the smallest estimated central black-hole mass from our Seyfert sample) $135 \mathrm{Myr}$ earlier, or from a $2.5 \times 10^{6} M_{\odot}$ black hole (the lower limit to the estimate of smallest visible quasars by the Wide survey at $z>6$ ) $166 \mathrm{Myr}$ earlier. In $\Lambda \mathrm{CDM}$, these correspond to redshifts $z=6.78$ and $z=7$, respectively, while in $R_{\mathrm{h}}=c t$, these times are associated with $z=6.51$ and $z=6.64$. Our minimum detectable mass estimate for the Medium-Deep survey is roughly $1.3 \times 10^{6} M_{\odot}$ in the middle of the redshift range for $\Lambda$ CDM. Thus, a $10^{8} M_{\odot}$ black hole at $z=6$ would have passed through this minimum at redshift $z \sim 7.22$ in $\Lambda \mathrm{CDM}$ and $z=6.76$ in $R_{\mathrm{h}}=c t$. Therefore, the predictions made in this paper are to be relied upon most strongly for $z>6.51$ in $R_{\mathrm{h}}=c t$, and $z>6.78$ for $\Lambda \mathrm{CDM}$, in the case of the Wide-field survey, and at $z>7$ and $z>7.22$, respectively, for the medium-deep survey.

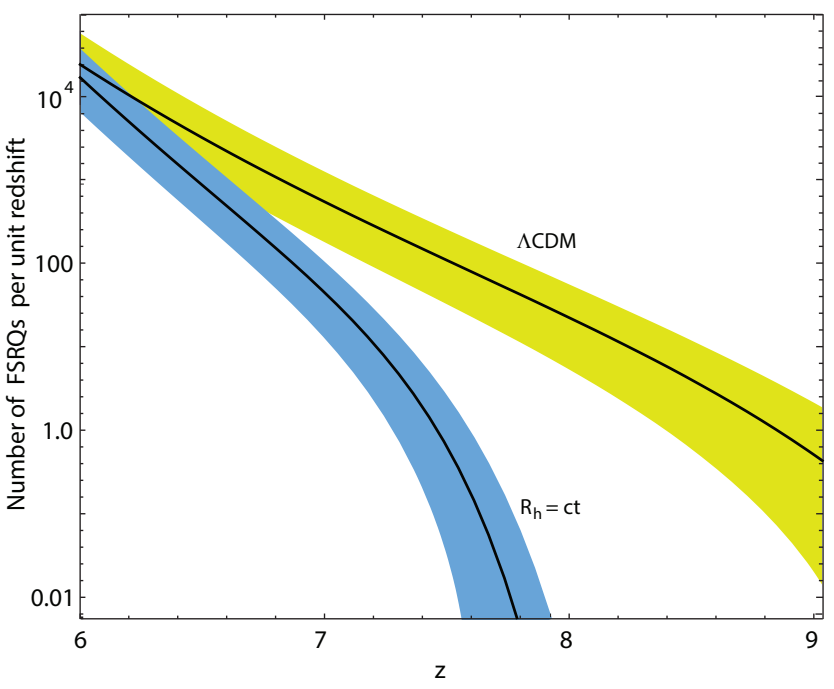

Figure 4. Estimated number of detected high-z FSRQs expected per unit redshift in the Wide survey, and our estimated error, for $R_{\mathrm{h}}=c t$ (blue swath) and $\Lambda \mathrm{CDM}$ (yellow swath).

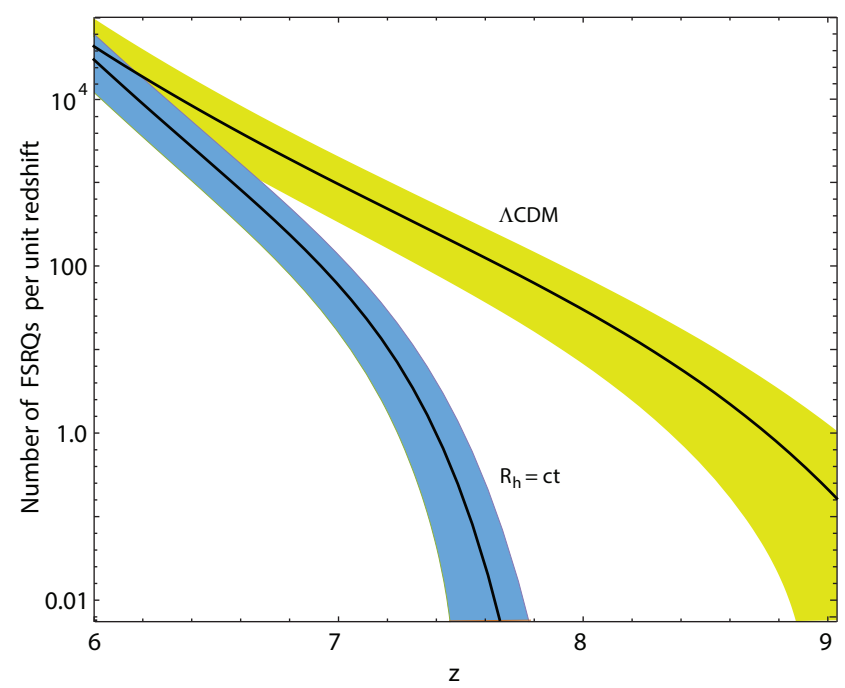

Figure 5. Estimated number of detected high-z FSRQs expected per unit redshift in the Medium-Deep survey, and our estimated error, for $R_{\mathrm{h}}=c t$ (blue swath) and $\Lambda$ CDM (yellow swath).

From here, we estimate the number of objects that would be visible over the whole sky within some redshift bin, using the integrated number

$N=\int_{z_{\text {low }}}^{z_{\text {high }}} d z^{\prime} \int_{\log _{10}\left[M_{\min }\left(z^{\prime}\right)\right]}^{\infty} \Phi\left(M, z^{\prime}\right) V_{z^{\prime}} d\left(\log _{10} M\right)$,

where $V_{z}=4 \pi d_{c}^{2} d / d z\left(d_{c}\right)$ is the differential comoving volume, and $M_{\min }(z)$ is the smallest detectable black-hole mass at redshift $z$. The function plotted in figures (4) and (5) is

$d N(z)=\int_{\log _{10}\left[M_{\min }\left(z^{\prime}\right)\right]}^{\infty} \Phi\left(M, z^{\prime}\right) V_{z^{\prime}} d\left(\log _{10} M\right)$.

The comoving distance, $d_{c}=d_{L} /(1+z)$, and volume are used based on the assumption that the number, $N$, of active galaxies remains constant in comoving volume regardless of redshift. Finally, we multiply this number by 0.0405 , based 
on the inferred fraction of flat-spectrum radio-loud quasars at $z>5.5$ (Bañados et. al. 2015; Coppejans et al. 2016), and then multiply it again by the fraction of sky covered by the survey ( 0.485 for the Wide survey and 0.121 for the Medium-Deep survey), to give a final estimate of the number of radio-loud objects expected to be visible with SKA. The results are presented in figures $4 \& 5$ and Tables 2 and 3 .

Included in each of these figures and tables are our estimated errors. In both cases, the Hubble constant acts as an inverse length scale that defines the comoving volume, which goes as $H_{0}^{-3}$. The primary source of uncertainty in our phenomenological model lies in the error associated with the smallest detectable black-hole mass. For example, when the best-estimate for a minimum mass is $10^{6} M_{\odot}$, the error from Equation (1) is \pm 0.28 mass dex, or up to $90 \%$. Since the Schechter mass function predicts more numerous smaller black holes, these variations can drastically alter the expected object counts. As such, we use the error in the central BH mass to adjust the lower limit of integration of Equations (10) and (11) in order to determine an upper and lower limit on the expected all-sky objects. This error is

$$
\delta N=\mp \int_{z_{\text {low }}}^{z_{\text {high }}} d z^{\prime} \int_{\log _{10}\left[M_{\min }\left(z^{\prime}\right)\right] \pm \delta \log _{10}\left[M_{\min }\left(z^{\prime}\right)\right]}^{\log _{10}\left[M_{\min }\left(z^{\prime}\right)\right]} \Phi\left(M, z^{\prime}\right) \times
$$

with the choice in signs corresponding to the upper and lower errors. We also include the errors in the high- $z$ radio-loud fraction (i.e., 8.1 $1_{-3.2}^{+5.0} \%$; Bañados et al. 2015) and the blazar fraction $(0.5 \pm 0.1)$ of those radio loud objects, based on the 26 objects in Coppejans et al. (2016). Added in quadrature, we find a final fractional error in the expected counts of

$\sigma_{N}^{+} \equiv\left(\frac{\delta N}{N}\right)^{2}+\left(\frac{0.1}{0.5}\right)^{2}+\left(\frac{0.05}{0.081}\right)^{2}$

at the upper end, and an analogous expression, $\sigma_{N}^{-}$(with 0.05 replaced with 0.032 ) at the lower end. These errors correspond to the colored swaths visualized in figures 4 and 5.

\section{DISCUSSION}

The results of this analysis suggest that SKA will detect a highly significant difference in the number of blazars as a function of redshift for the two cosmological models we have compared in this paper. We expect to find far more quasars in $\Lambda$ CDM than in $R_{\mathrm{h}}=c t$. The first few columns in Tables 2 and 3 point to the deficiencies in Willott et al.'s (2010) black-hole mass function at $z=6$. These suggest that SKA would be able to find nearly 10,000 radio-loud quasars in the redshift range $6 \lesssim z \lesssim 6.5$, roughly one per two square degrees in the wide survey. But the vast majority of these are due to an extrapolation of the $z=6$ mass function to masses far below those used to construct this distribution. With no real existing data to motivate the counts of smaller black holes at this redshift, these results have very little predictive power. Therefore, the predictions made in this paper are reliable only at $z \gtrsim 7$, as discussed in $\S \S$ III and IV.

The differences between the two models become more extreme with increasing redshift. At $z>7.22$, the standard $\Lambda \mathrm{CDM}$ model predicts that SKA will detect 80 times more blazars than $R_{\mathrm{h}}=c t$. The latter cosmology predicts effectively zero detections in either survey at $z>7.5$. The current most-distant known quasar of any type lies at a redshift $z=7.54$. This object has an estimated mass of $\sim 10^{8} M_{\odot}$ (Bañados et al. 2018). When we begin with Willot et al.'s black-hole mass function at $z=6$ and apply lockstep evolution, objects such as J1342+0928 appear to be statistically impossible for both considered cosmologies. But evidently this object and other similar objects at $z \gtrsim 7$ exist. Therefore, it becomes immediately clear that the simple evolution of the $z=6$ mass function does not accurately describe the full AGN population, if either of these cosmological models is to be believed. We point out, however, that the $z=6$ mass function does not take into account black holes that have already evolved past their primary active phase. Their host galaxies would then no longer appear active, and would be missed (or classified as some other kind of object if detected) by a survey. Therefore, we suggest that the $z=6$ mass function devolved to higher redshifts is a lower limit to the expected counts of existing AGNs. Our predicted numbers of FSRQs to be detected by SKA1 Wide and Medium Deep surveys thus represent lower limits on the number of objects that would be detected in either cosmological model.

Nonetheless, we point out that the number of objects predicted by $\Lambda$ CDM at $z>7$ in the Wide survey is more than a factor of 40 greater than the prediction by $R_{\mathrm{h}}=c t$. With the lower end of the $z>7$ estimate being 174, significantly above the $R_{\mathrm{h}}=c t$ range, we expect the results of the survey to strongly prefer one model over the other. Furthermore, $R_{\mathrm{h}}=c t$ predicts fewer than 1 detected blazar at $z>7.5$ in both surveys, while $\Lambda$ CDM still predicts $\sim 60$ in the wide survey, and $\sim 40$ in the medium-deep survey. Likewise, in the deep survey, $\Lambda$ CDM predicts roughly 100 blazars at $z>7.22$, while only $\sim 1$ is expected in $R_{\mathrm{h}}=c t$. We do not anticipate that quasars unaccounted for in our simplified evolution picture could overcome this large difference between the two models.

\section{CONCLUSION}

We have presented a method for predicting the number of Flat Spectrum Radio Quasars based on a phenomenological approach to estimating the radio luminosity of high-redshift sources. Using the observed population of AGNs with known $\gamma$-ray luminosities and mass estimates at lower redshifts, and using the Fermi Blazar Sequence, we have constructed a broad-band spectral energy distribution of FSRQs as a function of mass alone. We have then used this spectrum to estimate the smallest black-hole mass of a FSRQ detectable by SKA as a function of redshift. Finally, we have devolved the black-hole mass function observed at $z=6$ to higher redshifts, assuming lockstep evolution.

With the assumption that all the black holes above the limiting mass at a given redshift would be visible to a complete flux-limited survey, we have predict the number counts of FSRQs expected as a function of redshift for two competing models, $\Lambda \mathrm{CDM}$ and $R_{\mathrm{h}}=c t$. Taking into account the limitations used to construct the black-hole mass function at $z=6$, we have predicted the counts of objects at $z>7$ for the wide-field SKA band 1 survey, and at $z>7.22$ in the medium-deep SKA band 2 survey. These results offer a 
Table 2. Estimated counts of objects to be detected by the SKA1 Wide Band 1 survey. Note the anomalously high expected detections for $z<6.5$. This is due to the BH Mass function at $z=6$ being extrapolated to lower masses. The two rightmost columns present the estimates for $z$ above the redshifts for BHs in $\Lambda \mathrm{CDM}$ to have devolved such that a BH at $z=6$ is smaller than the smallest known gamma-ray Radio-loud Seyfert 1 by $z=6.78$, and below the inferred lower limit for detectable BHs at $z=7$, respectively.

\begin{tabular}{|c|c|c|c|c|c|c|c|c|}
\hline Redshift Range $(z)$ : & $6-6.5$ & $6.5-7$ & $7-7.5$ & $7.5-8$ & $8-8.5$ & $8.5-9$ & $6.78-9$ & $7-9$ \\
\hline$\Lambda \mathrm{CDM}$ & $9881_{-6193}^{+10117}$ & $1492_{-945}^{+1545}$ & $271_{-178}^{+296}$ & $51_{-36}^{+64}$ & $8.0_{-6.5}^{+13.5}$ & $0.80_{-0.75}^{+2.33}$ & $694_{-454}^{+757}$ & $330_{-221}^{+375}$ \\
\hline$R_{h}=c t$ & $5178_{-3089}^{+4785}$ & $273_{-172}^{+273}$ & $8.1_{-6.3}^{+12.4}$ & $0.01_{-0.01}^{+0.08}$ & 0 & 0 & $45_{-31}^{+54}$ & $8.1_{-6.3}^{+12.4}$ \\
\hline
\end{tabular}

Table 3. Estimated counts of objects to be detected by the SKA1 Medium-Deep Band 2 survey. Redshift bins are the same as the Table 2 , except the rightmost bin covers $z>7.22$.

\begin{tabular}{|c|c|c|c|c|c|c|c|c|}
\hline Redshift Range $(z)$ : & $6-6.5$ & $6.5-7$ & $7-7.5$ & $7.5-8$ & $8-8.5$ & $8.5-9$ & $6.78-9$ & $7.22-9$ \\
\hline$\Lambda \mathrm{CDM}$ & $5107_{-3341}^{+5768}$ & $786_{-517}^{+891}$ & $151_{-101}^{+176}$ & $32_{-23}^{+41}$ & $6.6_{-5.2}^{+10.3}$ & $1.1_{-1}^{+2.5}$ & $385_{-259}^{+452}$ & $96_{-67}^{+120}$ \\
\hline$R_{h}=c t$ & $2706_{-1688}^{+2741}$ & $157_{-101}^{+167}$ & $7.2_{-5.4}^{+10.3}$ & $0.05_{-0.05}^{+0.23}$ & 0 & 0 & $31_{-22}^{+38}$ & $1.2_{-1.1}^{+2.5}$ \\
\hline
\end{tabular}

definitive method of discriminating between these two cosmological models, in which $\Lambda$ CDM predicts 30 times more blazar counts than $R_{\mathrm{h}}=c t$ in the wide survey, and 80 times more in the medium-deep survey.

While there appear to be other influences at work regarding the evolution of the black-hole mass at high redshifts, this analysis may be viewed as providing a lower limit to the overall number of such sources expected to be seen by SKA at those redshifts. At this time, we do not anticipate any additional corrections in $R_{\mathrm{h}}=c t$ that could reasonably explain the detection of $\sim 200$ such sources by the Wide survey at $z>7$ if this is what SKA finds. But if fewer than $\sim 50$ detections are made by the time the survey is completed, it would correspondingly be very difficult to explain this deficit in the context of the standard model, thereby strongly favouring $R_{\mathrm{h}}=c t$.

We also point out that the phenomenological approach used in this paper may be applied to any other nonthermal waveband of interest, allowing for predictions of FSRQ-type objects by different surveys in other parts of the spectrum. In addition, the SKA surveys are poised to discover AGNs with smaller black-hole masses than ever observed before at $z>6$. We expect that SKA detections at $z \sim 6$ will thereby extend the $z=6$ black-hole mass function to significantly lower masses, thus permitting us to eventually devolve this improved mass distribution towards higher redshifts. Our ultimate goal is to form a sufficiently complete picture of AGN growth in the early universe in order to convincingly rule out one or other of these two models at a very high level of confidence.

\section{ACKNOWLEDGMENTS}

We thank Jinyi Yang, Xiaohui Fan, and Feige Wang for helpful discussions concerning recent measurements of highredshift quasars.

\section{REFERENCES}

Ackermann M., et al., 2015, VizieR Online Data Catalog, p. J/ApJ/810/14

An H., Romani R. W., 2018, ApJ, 856, 105

Antonucci R., 1993, ARA\&A, 31, 473

Bañados E., et al., 2015, ApJ, 804, 118

Bañados E., et al., 2018, Nat, 553, 473

Beckmann V., Shrader C. R., 2012, Active Galactic Nuclei

Bull P., et al., 2018, arXiv e-prints, p. arXiv:1810.02680

Coppejans R., et al., 2016, MNRAS, 463, 3260

Fabian A. C., Walker S. A., Celotti A., Ghisellini G., Mocz P., Blundell K. M., McMahon R. G., 2014, MNRAS, 442, L81

Fan X., et al., 2001, AJ, 122, 2833

Fatuzzo M., Melia F., 2017, ApJ, 846, 129

Ghisellini G., Sbarrato T., 2016, MNRAS, 461, L21

Ghisellini G., Maraschi L., Tavecchio F., 2009a, MNRAS, 396, L105

Ghisellini G., Foschini L., Volonteri M., Ghirlanda G., Haardt F., Burlon D., Tavecchio F., 2009b, MNRAS, 399, L24

Ghisellini G., Tavecchio F., Foschini L., Ghirlanda G., Maraschi L., Celotti A., 2010, MNRAS, 402, 497

Ghisellini G., Righi C., Costamante L., Tavecchio F., 2017, MNRAS, 469, 255

Hovatta T., Valtaoja E., Tornikoski M., Lähteenmäki A., 2009, A\&A, 494, 527

Krolik J. H., Begelman M. C., 1986, ApJ, 308, L55 
Leaf K., Melia F., 2017, MNRAS, 470, 2320

Lusso E., Risaliti G., 2016, ApJ, 819, 154

Melia F., 2003, The Edge of Infinity

Melia F., 2007, MNRAS, 382, 1917

Melia F., 2013, ApJ, 764, 72

Melia F., 2016, Frontiers of Physics, 11, 119801

Melia F., 2017, MNRAS, 464, 1966

Melia F., 2018, MNRAS, 481, 4855

Melia F., Abdelqader 2009, International Journal of Modern Physics D, 18, 1889

Melia F., Koenigl A., 1989, in Hunt J., Battrick B., eds, ESA Special Publication Vol. 296, Two Topics in X-Ray Astronomy, Volume 1: X Ray Binaries. Volume 2: AGN and the X Ray Background. pp 995-1000

Melia F., Maier R. S., 2013, MNRAS, 432, 2669

Melia F., McClintock T. M., 2015, Proceedings of the Royal Society of London Series A, 471, 20150449

Melia F., Shevchuk A. S. H., 2012, MNRAS, 419, 2579

Melia F., Yennapureddy M. K., 2018a, MNRAS, 480, 2144

Melia F., Yennapureddy M. K., 2018b, Journal of Cosmology and Astro-Particle Physics, 2018, 034

Paliya V. S., Stalin C. S., Shukla A., Sahayanathan S., 2013, ApJ, 768,52

Paliya V. S., Ajello M., Rakshit S., Mand al A. K., Stalin C. S., Kaur A., Hartmann D., 2018, ApJ, 853, L2

Paliya V. S., Parker M. L., Jiang J., Fabian A. C., Brenneman L., Ajello M., Hartmann D., 2019, ApJ, 872, 169

Planck Collaboration et al., 2018, arXiv e-prints, p. arXiv:1807.06209

Riess A. G., et al., 2018, ApJ, 861, 126

Shakura N. I., 1973, Soviet Ast., 16, 756

Sikora M., Madejski G., 2001, in Aharonian F. A., Völk H. J., eds, American Institute of Physics Conference Series Vol. 558, American Institute of Physics Conference Series. pp 275-288 (arXiv: astro-ph/0101382), doi:10.1063/1.1370797

Square Kilometre Array Cosmology Science Working Group et al., 2018, arXiv e-prints,

Urry C. M., Padovani P., 1995, PASP, 107, 803

Volonteri M., Haardt F., Ghisellini G., Della Ceca R., 2011, MNRAS, 416, 216

Wang F., et al., 2018, arXiv e-prints, p. arXiv:1810.11926

Weigel A. K., Schawinski K., Treister E., Urry C. M., Koss M., Trakhtenbrot B., 2015, MNRAS, 448, 3167

Willott C. J., et al., 2010, AJ, 140, 546

Wu J., Ghisellini G., Hodges-Kluck E., Gallo E., Ciardi B., Haardt F., Sbarrato T., Tavecchio F., 2017, MNRAS, 468, 109

Yennapureddy M. K., Melia F., 2018, European Physical Journal C, 78, 258

This paper has been typeset from a $\mathrm{T}_{\mathrm{E}} \mathrm{X} / \mathrm{LAT}_{\mathrm{E}} \mathrm{X}$ file prepared by the author. 